\title{
Design of a Fuzzy Controller for the Automation of an Extracorporeal Support System with the use of a Simulation Environment
}

\author{
A. Mendoza G., B. Baumgartner, U. Schreiber, S. Eichhorn, M. Krane, R. Bauernschmitt, A. Knoll
}

\begin{abstract}
The automation of a portable extracorporeal support system may greatly help people who suffer from cardiogenic shock by providing them with an optimal oxygen perfusion and avoid mult-organ failure while being transported to a hospital. This however requires the creation of tools that help in the design of the ideal controller. In this paper a simulation environment is described were a cardiovascular model from the ISR Physiome database was used together with a model of a portable extracorporeal support system. Additionally a model of an oxygenator/blender model is introduced to allow the simulation of oxygen perfusion. Fuzzy controlling was used for automation since it allows a straightforward implementation of expert knowledge. Through the simulation environment different scenarios may be created where intensive testing is possible and constant repetition for control optimization. Initial simulation results are given of the fuzzy controller adjusting the extracorporeal flow rate and oxygen administration for a case of low cardiac output.
\end{abstract}

\section{INTRODUCTION}

The development of new technologies have enabled the miniaturization of medical devices, allowing them to be used in new areas where portability is necessary. This gives great benefits to the patient since medical treatment may be given faster in the place needed. A specific case is the use of an extracorporeal support system (ECSS). These systems are routinely used in cardiac surgery [1], however patients who suffer from a cardiogenic shock can benefit with the use of a portable ECSS reducing the risk of death or multi-organ failure [2], [3], [4]. This brings new challenges since the current operation of an ECSS is made by trained perfusionists who may not always be available on site. For this reason the objective of this project is to design a controller capable of monitoring the patients vital signals and adjust the ECSS parameters reducing the workload of a human operator while giving an optimal perfusion to the patient.

In order to design this controller a simulation environment was created capable of recreating the behavior of the cardiovascular system connected to an ECSS. Previous work was presented where a cardiovascular model from the NSR Physiome Project was extended with a model of a portable heart-lung machine (HLM) [5]. In the current paper a description is given of how the previous model was extended to simulate the gas exchange happening inside the lungs and the gas exchange within the Oxygenator of the

A. Mendoza G., B. Baumgartner and A. Knoll are with the Robotics and Embedded Systems, Faculty of Informatics, Technische Universität München, 85748 Garching, Germany,

U. Schreiber, S.Eichhorn, M. Krane and R. Bauernschmitt are with the Cardiovascular Surgery, German Heart Center Munich, Technische Universität München, 85748 Garching, Germany, Email:mendozagein.tum.de
HLM. This allows the regulation of oxygen concentration given to the oxygenator with the use of a gas blender. This regulation will depend on the blood oxygen saturation $(\mathrm{SpO} 2)$ of the patient and the extracorporeal flow rate (EFR).

As a control mechanism fuzzy logic was used since it gives the possibility of translating the experience of doctors to rules that can be easily implemented by the controller.

To allow the simulation of different scenarios the model was modified to represent various patient disorders. Additionally a fuzzy control is described as a multiple input multiple output (MIMO) system, controlling the speed of the centrifugal pump and the fraction of inspired oxygen (FiO2) together with the gas volume provided by the oxygen blender.

At the end results are given of the control behavior during simulation. The use of this simulation environment has proven to be appropriate in the process of designing a controller to be used in an ECSS.

\section{Extracorporeal Circulation}

When a patient suffers from cardiogenic shock its heart is not capable of generating enough blood flow to maintain adequate tissue perfusion. This bring complications increasing the risk of multi-organ failure and possible death. Proper treatment can be given to the patient within a hospital however the risk of death is increased in the absence of oxygen perfusion to the body while being transported. To overcome this a portable ECSS is used. This device circulates the blood out of the patient to be reoxigenated in an oxygenator and is sent back to the body. Its main components are a centrifugal pump, a gas oxygenator a reservoir and a filter. The blood flow generated by the centrifugal pump and the amount of oxygen administered is adjusted by a trained perfusionist. For the proper operation of the ECSS constant supervision and adjustemnts are required, however with the use of a controller the need of constant supervision may be reduced, focusing the atention on the patient.

\section{Simulation Model}

A simulation model was used to design and test the designed controller. This model consists of two main components: a cardiovascular model and an ECSS model describing the extracorporeal blood flow and blood oxygenation.

\section{A. Cardiovascular Model}

The cardiovascular system was based on a closed-loop model obtained from the ISR Physiome database [6]. It consists of a four-chamber varying-elastance heart, a pericardium, a systemic circulation and pulmonary circulation. 

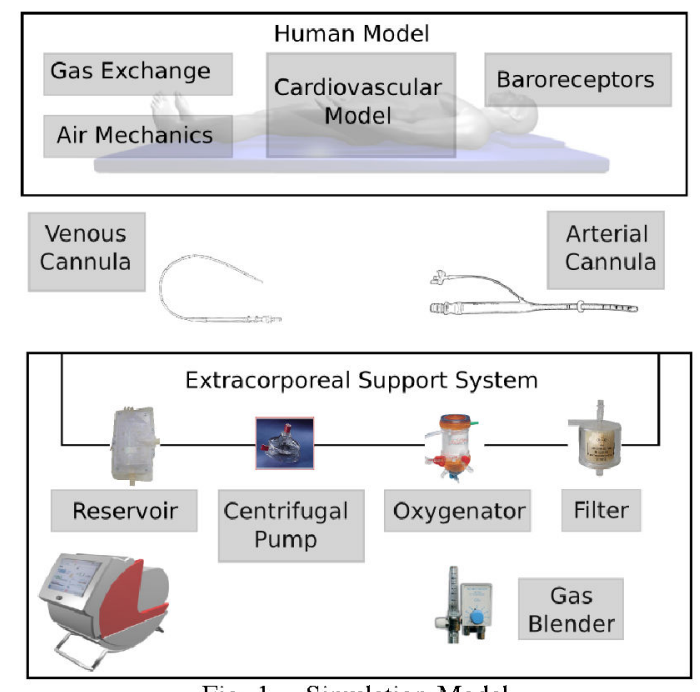

Fig. 1. Simulation Model

A baroreceptor model is used to control the heart-rate. The air mechanics and gas exchange model were included to calculate the oxygen absorbed by the lungs and consumed by the body together with the carbon dioxide produced by the body and extracted through the lungs. More information related to this may be found at [7], [8], [9], [10].

\section{B. ECSS Model}

An existing HLM [11] was used as a reference for the model creation of an ECSS system. This was done by analyzing the resistance behavior of all of its individual elements (reservoir, centrifugal pump, oxygenator, filter, tubing, venous cannula and arterial cannula). A quadratic function was used to describe the difference in pressure in terms of the flow going through each component:

$$
\triangle P_{i}=C a_{i} Q^{2}+C b_{i} Q
$$

The values for $C a$ and $C b$ of each component may be obtained from [5]. The resulting flow generated by the HLM may by calculated given the following formula:

$$
\begin{gathered}
P_{S}(r p m)-\frac{\Delta P_{C V M}}{\rho g}= \\
\left(\frac{\sum_{i=1}^{7} C a_{i}}{\rho g}+\frac{1}{2 A^{2} g}\right) Q^{2}+ \\
\left(\frac{\sum_{i=1}^{7} C b_{i}}{\rho g}-P_{D}(r p m)\right) Q
\end{gathered}
$$

Where $Q$ is the resulting flow, $P_{S}$ and $P_{D}$ are factors obtained depending on the rpm of the centrifugal pump and $\triangle P_{C V M}$ is the difference of pressure obtained from the input and output of the cardiovascular model. $A$ is the pump output area, $\rho$ is the blood density and $g$ is gravity.

\section{Oxygenator}

Systemic oxygen delivery is one of the most important determinants of optimal perfusion when using an ECSS. It will permit the body organs to receive the needed oxygen and extract the produced carbon dioxide.

In the ECSS a membrane oxygenator is used for this purpose. The gas exchange between the oxygenator and the blood is determined by the difference of the gas partial pressures and its corresponding diffusion factor [12]. Formula 2 shows the change of partial pressure of a gas over time.

$$
\frac{d P_{G}}{d t}=\frac{\dot{V}_{G} \cdot\left(P_{G, i}-P_{G}\right)+P_{a t m} \cdot D_{G} \cdot\left(\operatorname{Blood} P_{G, i}-P_{G}\right)}{V_{G}}
$$

The subindex ${ }_{G}$ corresponds to either $\mathrm{O}_{2}$ or $\mathrm{CO}_{2} . \dot{V}_{G}$ is the flow of the gas mixture introduced into the oxygenetor and is determined by the oxygen blender. $P_{G, i}$ is the input partial pressure of $\mathrm{O}_{2}\left(\mathrm{PO}_{2}\right)$ and $\mathrm{CO}_{2}\left(\mathrm{PCO}_{2}\right)$. The input of $\mathrm{PCO}_{2}$ is $0 \mathrm{mmHg}$. The input $\mathrm{PO}_{2}$ is determined by the oxygen blender obtained by multiplying $\mathrm{FiO}_{2}$ times the atmospheric pressure $P_{a t m}(760 \mathrm{mmHg}) . D_{G}$ is the diffusion factor for $\mathrm{O}_{2}$ and $\mathrm{CO}_{2}$. Blood $P_{G, i}$ corresponds to the gas partial pressure of the blood entering the oxygenator. This is obtained from the gas partial pressures at the pulmonary artery from the gas exchange model of the cardiovascular system.

\section{Patient disorders}

Several parameters were used in the model to represent the behaviour of a patient with different disorders. With this parameters the heart-rate may be increased or decreased with the possibility of a complete stop and arrhythmia. Additionally it is possible to simulate a malfunction of the mitral valve, causing a back flow between the left atrium and left ventricle of the heart, decrease the elastance from the right or left ventricle producing less cardiac output, and change the respiration frequency. In the ECSS it is possible to simulate leakage from a bad connection or the clamping of the arterial/venous tubing connecting the ECSS and the patient.

\section{FUZZY CONTROL}

A standard fuzzy system with Mamdami inference and center of gravity was used to implement the controller. Previous work of the fuzzy control is described at [13]. This was extended to improve the control response.

\section{A. Input Variables}

The input variables were selected for the controller corresponding to signals that are meassured in a real system with the use of different sensors. These signals are: the mean arterial pressure (MAP) which can be obtained directly from the arterial cannula with a pressure transducer, the extra corporal flow rate (EFR) captured with an ultrasonic flow probe within the ECSS and the oxygen saturation (SpO2) captured with a non invasive oxymeter or a gas analyzer for more precise measurements.

For each input a target value may be set. These values will depend on the patients precondition. As an example patients suffering from atheriosclerotic disease of the aorta 


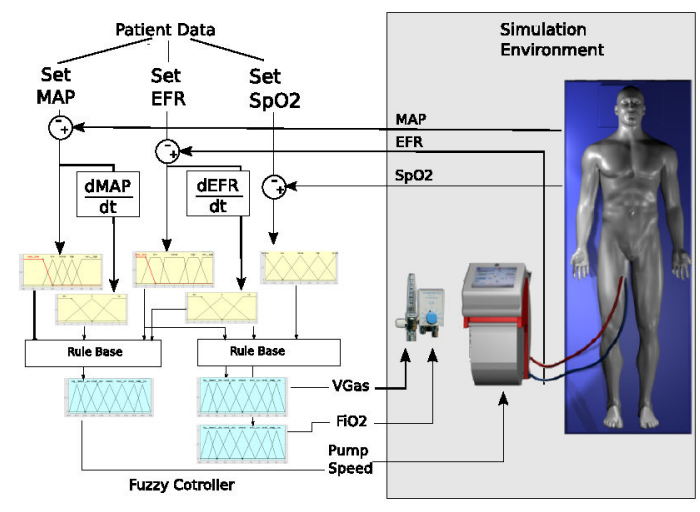

Fig. 2. Controller

or patients with diabetes may require a higher blood pressure as described in [14]. For this reason previous knowledge of the patient history may help improve the initial settings. Once the values of each input are set the difference between the target value and the patient signal is obtained. These differences were introduced as inputs and were defined in the controller with 5 membership functions defined as "Very High", "High", "Normal", "Low" or "Very Low". Additionally the derivative of the MAP and EFR was used as input and 3 membership functions were used to describe this, defined as "Increasing", "Steady" and "Decreasing".

\section{B. Output Variables}

The parameters that are changed during perfusion are the centrifugal pump speed, the oxygen $\mathrm{FiO} 2$ and the amount of gas volume given to the oxygenator. These parameters are controlled by the output of the controller with the use of 7 membership functions defined as "Big Increase", "Medium Increase", "Small Increase", "Steady", "Small Decrease", "Medium Decrease" and "Big Decrease". For each value maximum and minimum limits were defined to stay within possible values.

\section{Rule Base}

Two rule sets were created, the first one controlling the centrifugal pump speed receiving as an input the $M A P$, $d M A P / d t, E F R$, and $d E F R / d t$. The second rule set was used to control the FiO2 and gas volume where the $E F R$, $d E F R / d t$ and $S p O 2$ were set as inputs.

\section{IMPLEMENTATION}

A Mathematical Modeling Language (MML) was used to describe the model which is then compiled and executed by the simulation system called JSim [15].

With JSim it is not possible to do a continuous simulation and the control values may not be introduced on runtime. For this reason a simulation engine program was created which takes control of the JSim simulation system (Figure 3).

A simulation period is selected defined as the time JSim will calculate the simulation values. Additionally a time step is introduced for the number of values to calculate over the given period. To have a continuous simulation state variables are used were initial values are introduced to the simulation, after execution the state variables are recovered and used for the next simulation iteration. Before starting an iteration new input values are introduced into the simulation and when finished output values are obtained.

Specific output variables representing the patient vital signals are sent to a server application through a TCP socket connection. The fuzzy controller was executed in a second computer since the simulation model requieres intensive processing. This computer connects to the server application to receive the simulation outputs which are used as control inputs.

For test purposes the controller was designed in Matlab with the use of the Fuzzy Logic Toolbox [16] allowing the visualization of the different rules active at a specific time of simulation. This allows the verification of contradictory rules and elimination of unused rules. Once the control outputs are calculated they are sent back to the simulation engine to be introduced in the next simulation iteration as inputs.

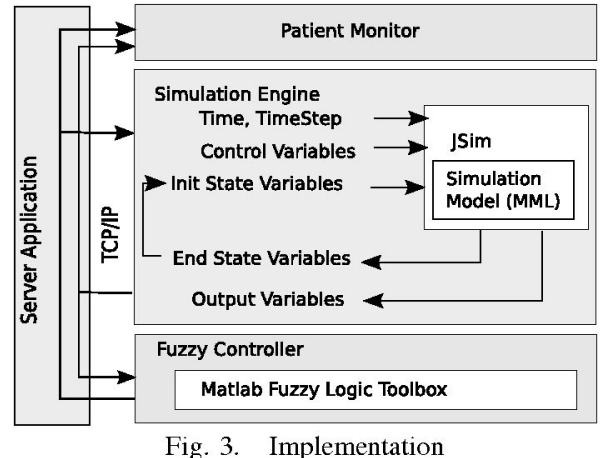

\section{RESULTS}

Initial parameters were given to the model to simulate a patient with a low MAP and a heart with a low cardiac output due to a bad mitral valve and a reduced ventricle elastance. The target MAP was set to $60 \mathrm{mmHg}$ and an EFR of 4 $\mathrm{L} / \mathrm{m}$. Figure 4 shows the results of this simulation. After reaching the target MAP this was changed to $50 \mathrm{mmHg}$ to test the controller behavior. This target is not completely reached since the rule base of the speed control was set to not decrease the pump speed if this causes a reduction of the EFR. After 800 seconds the target MAP was increased to $70 \mathrm{mmHg}$. The controller was able to achieve this target however the centrifugal pump reached its maximum speed.

The two last graphs of figure 4 show the results of the oxygen blender control. The $\mathrm{SpO} 2$ target was set to $87 \%$. Since the model has a lower $\mathrm{SpO} 2$ the $\mathrm{FiO} 2$ and Vgas is set to a maximum value. When reaching the target Spo2 both the FiO2 and Vgas were decreased to keep a stable saturation. When a different target for the MAP is selected the blender control automatically adjusts to these settings. For the case of reducing the MAP this decreases the EFR causing the controller to increase the oxygen administration to the oxygenator. In the case of the higher blood pressure the FiO2 and gas volume is reduced due to a higher EFR. 

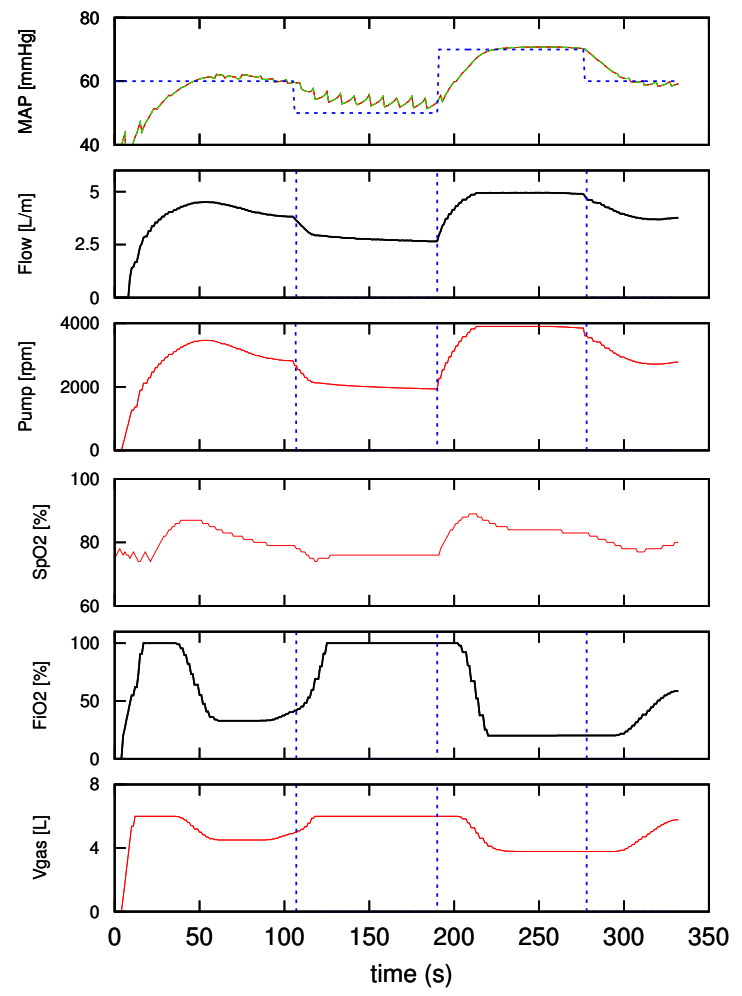

Fig. 4. Simulation Results

\section{DISCUSSION}

A simulation environment has proven to be essential in the development of a controller for a medical device. It allows the verification of the controller software components by generating different scenarios and constant repetition thus eliminate the risk of failure. Additionally the capability of stopping a simulation at a given time and analyzing a specific setup step by step allows the correction and optimization of the implemented rules.

Previous work [13] reported the results of using a simple fuzzy controller. By adding more membership functions to the input and output variables and including the input tendencies it was possible to model the behavior of the controller in more detail. The reaction of the output variables may be adjusted when the target values are further away from the inputs and also when the target value is almost reached avoiding an overshoot.

\section{CONCLUSIONS AND FUTURE WORKS}

\section{A. Conclusions}

A portable ECSS may be of great benefit for people who suffer from cardiogenic shock. It provides oxygen perfusion essential to the body while being transported to a hospital. To achieve this a constant and proper adjustment of the ECSS is needed. This is made possible with the automation of the ECSS. Fuzzy control allowed a straight forward implementation of expert knowledge.

Intensive tests are needed for the verification of the controller. With the simulation environment a detailed analysis of the implemented rules can be done by constantly recreating different scenarios until an optimal control response is achieved.

\section{B. Future Works}

Including more variables and increasing the membership functions for each input increases significantly the amount of rules needed. A learning algorithm is to be designed to help in the creation of the required rules and the adjustment of the membership functions. Additionally this learning algorithm is intended to adapt to the needs of each individual patient with the implementation of a neuro-fuzzy controller.

\section{REFERENCES}

[1] G. P. Gravlee, R. F. Davis, A. H. Stammers, and R. M. Ungerleider, Cardiopulmonary Bypass: Principles and Practice. Wolters Kluwer, 2008.

[2] O. Maunz, J. Horisberger, and L. von Segesser, "Bridge to life: The lifebridge b2t coextracorporeal life support system in an in victro trial," Perfusion, vol. 23, pp. 279-282, 2008.

[3] M. Uwe Mehlhorn, D.-I. Michael Brieske, M. Uwe M. Fischer, M. Markus Ferrari, M. Patrick Brass, M. Juergen H. Fischer, and M. Hans-R. Zerkowski, "Lifebridge: A portable, modular, rapidly available plug-and-play mechanical circulatory support system," The Annals of Thoracic Surgery, vol. 80(5), pp. 1887-1892, 2008.

[4] M. Krane, D. Mazzitelli, U. Schreiber, A. Mendoza, S. Braun, B. Voss, C. Badiu, G. Brockmann, R. Lange, and R. Bauernschmitt., "Lifebridge b2t a new portable cardiopulmonary bypass system." ASAIO J, vol. 56, pp. 52-56, 2010.

[5] A. Mendoza Garcia, B. Baumgartner, U. Schreiber, M. Krane, A. Knoll, and R. Bauernschmitt, "Simulation of extracorporeal circulation for the design of a fuzzy controlled perfusion," Seventh IASTED conference Biomedical Engineering, 2010.

[6] "Physiome at http://nsr.bioeng.washington.edu." [Online]. Available: http://nsr.bioeng.washington.edu

[7] M. Hassan, M. El-Brawany, and M.M.Sharaf, "A functional cardiovascular model with disorders," Engineering in Medicine and Biology, vol. 27, pp. 5089-5092, 2005.

[8] K. Lu, J. C. Jr., F. Ghorbel, D. Ware, and A.Bidani, "A human cardiopulmonary system model applied to the analysis of the valsava maneuver," Physiol Heart Circ, vol. 281, pp. 2661-2679, 2001.

[9] R. Bauernschmitt, E. Naujokat, H. Mehmanesh, S. Schulz, C. Vahl, S. Hagl, and R. Lange, "Mathematical modeling of extracorporeal circulation: Simulation of different perfusion regimens perfusion," Perfusion, vol. 14, pp. 321-330, 1999.

[10] C.Luo, D. Ware, J. Zwischenberger, and J. C. Jr., "Using a human cardiopulmonary model to study and predict normal and diseased ventricular mechanics, septal interaction, and atrio-ventricular blood flow patterns," Cardiovascular Engineering, vol. 7:17, p. 31, 2007.

[11] M. Krane, D. Mazzitelli, U. Schreiber, A. M. Garcia, B. Voss, C. Badiu, R. Lange, and R. Bauernschmitt, "First experience with a new portable cardiopulmonary bypass system - lifebridge b2t with percutaneous femoral cannulation," Comp. In Cardiology, pp. 269272, 2008.

[12] G. Meyrowitz, Automatisierung der Herz-Lungen-Maschine. Mensch \& Buch Verlag, 2005, ch. 7, p. 179.

[13] B. Baumgartner, A. Mendoza Garcia, U. Schreiber, S. Eichhorn, M. Krane, R. Bauernschmitt, and A. Knoll, "A simple fuzzy controller for an extra-corporeal circulation system - limitations and potentials." 5th Russian Bavarian Congress Proceedings, 2009.

[14] M. Glenn S. Murphy, M. Eugene A. Hessel II, and M. Robert C. Groom, "Optimal perfusion during cardiopulmonary bypass: An evidence-based approach," Anesthesia Analg., vol. 108(5), pp. 13941417, 2009.

[15] "Jsim at http://nsr.bioeng.washington.edu/jsim/." [Online]. Available: http://nsr.bioeng.washington.edu/jsim/

[16] "Fuzzy logic toolbox at http://www.mathworks.com/products/fuzzylogic/." 